\title{
Ionometric Half Value Layer Meter for Measurements of the Quality of X-Rays*
}

\author{
By R. H. Herz \\ Research Laboratories, Kodak Ltd., Wealdstone, Harrow, Middx. (England) \\ (Z. Naturforschg. 6 a, 350-352 [1951]; eingegangen am 15. Mai 1951) \\ To Mr. John Eggert for his $60^{\text {th }}$ birthday
}

\begin{abstract}
An instrument is described which permits the measurement of the quality of X-rays in terms of the half value layer (HVL) of a suitable filter material. It consists of two ionization chambers adjustable in volume, which are connected in opposition and linked with an X-ray dose measuring device. The chambers are so adjusted that one chamber is twice as sensitive as the other. The filtration of the $\mathrm{X}$-ray beam entering the larger chamber is adjusted until a balance position is reached as indicated by zero deflection of the electrometer needle of the measuring device.
\end{abstract}

$T_{x}$ he determination of the quality of a heterogeneous $\mathrm{X}$-ray beam is an essential requirement in most quantitative investigations on the photographic action of X-rays, as the sensitivity of photographic material depends on the X-ray wavelength applied. The beam emitted by an X-ray tube is characterized by a wide spectrum of wavelengths and several methods have been employed in order to estimate the photographically effective mean wavelength of an X-ray beam. Such methods consist of a spectral analysis of the beam by means of an X-ray spectrograph, of a measurement of the varying potential across the electrodes of the X-ray tube or of an estimate of the penetrating power of a beam by means of a filter analysis. In this paper a simplified version of the latter method is suggested which has proved to be suitable and convenient in research work of the kind indicated above.

\section{Analysis by filters and half value layer ${ }^{1-8}$}

The percentage transmission of the flux of X-rays through a filter of a given material and thickness is directly related to the quality. The quality of a heterogeneous beam is commonly referred to by the half value layer (HVL) and this is defined as that

* Communication No. $1406 \mathrm{H}$ from the Kodak Research Laboratories.

1 L. S. T a y lor and G. S i n g e r, Radiology 22, 4 445 [1934]

2 L. S. T a y lor, G. S inger and C. F. S to neb u rn e r, Bur. Standards J. Res. 11, 2, 292 [1933].

3 W. B i n k s, Brit. J. Radiol. 9, 107 [1936].

4 R. B. Wils e y, Radiology 17, 700 [1931]. thickness of a given filter material which reduces the flux of the incident radiation by half.

Although the HVL may be used to determine the quality of a heterogeneous X-ray beam, it does not necessarily follow that X-ray beams having the same HVL have the same wavelength distribution. For any heterogeneous X-ray beam, it is.found that if it is progressively filtered, its HVL increases with increasing filtration up to a filtration at which the $\mathrm{X}$-ray beam becomes almost homogeneous. The practical procedure for determining the HVL can be carried out for instance, by interposing increasing filter thickness of the same material, say copper, between the X-ray tube and an ionization chamber. By plotting the logarithm of the dosage rate of X-rays transmitted against the thickness of the absorber a logarithmic absorption curve is obtained. The HVL is then found by interpolation of that thickness of filter which has reduced the initial dosage rate by 50 per cent. The slope of the absorption curve decreases with increasing filtration, since the radiation suffers differential absorption for the rays of different wavelengths present, the softer components of the $\mathrm{X}$-ray beam being more readly absorbed.

The procedure for determining the HVL as described above is laborious, as many successive

5 E. D. Trout and Z. J. Atlee, Amer. J. Roentgenol. 47, 5, 785 [1942].

${ }_{6}^{6}$ W. V. M a y n e ord and J. E. Ro bert s, Brit. J. Radiol. 8, 41 [1935].

7 L. A. W. K e m p, Brit. J. Radiol. 19, 233 [1946].

$8 \mathrm{H}$. Holthusen u. R. B ra un, Grundlagen und Praxis der Röntgenstrahlen-Dosierung. Gg. Thieme, Leipzig 1933. 
absorption measurements have to be made. It also suffers from the disadvantage of possible fluctuations of the mains supplying the $\mathrm{X}$-ray unit in consecutive measurements which may influence the shape of the absorption curve. In order to overcome the difficulties mentioned above a special half value layer meter has been constructed, the design of which is described in the following section.

Construction of half value layer meter Basis principle

The apparatus (see circuit Fig. 1) consists of an $\mathrm{X}$-ray dosemeter of almost conventional design which

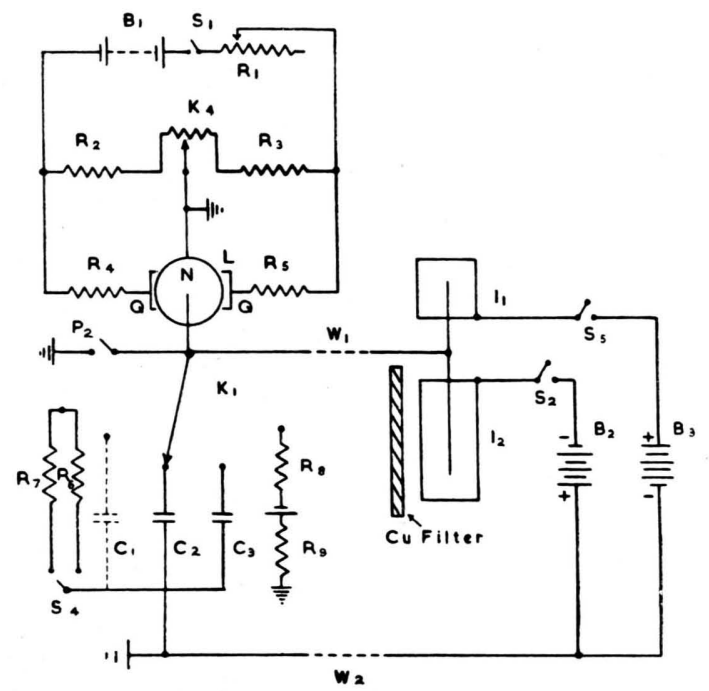

Fig. 1. Circuit of HVL meter. $B_{1}, 54$ volt battery for electrometer quadrant potential; $\mathrm{B}_{2}, \mathrm{~B}_{3}, 54$ volt batteries for chamber potentials; $R_{1}$, variable resistance for adjusting sensitivity of Lindeman electrometer; $\mathrm{R}_{2}, \mathrm{R}_{3}, 75,000 \Omega$; $\mathrm{R}_{4}, \mathrm{R}_{5}, 100,000 \Omega ; \mathrm{R}_{6}, 10,000 \mathrm{M} \Omega ; \mathrm{R}_{7}, 1,000 \mathrm{M} \Omega ; \mathrm{R}_{8}$, $\mathrm{R}_{\mathbf{9}}, 100,000 \Omega$; L, Lindeman electrometer; N, Electrometer needle; Q, Quadrants; $\mathrm{K}_{4}$, Potentiometer for balance ccntrol of needle; $\mathrm{K}_{1}$, High insulating switch; $\mathrm{C}_{1}$, Cable capacity; $\mathrm{C}_{2}, 0,001 \mu \mathrm{F} ; \mathrm{C}_{3}, 0,01 \mu \mathrm{F} ; \mathrm{C}$, Weston standard cell; $\mathrm{P}_{2}$, Earthing switch; $\mathrm{I}_{1}, \mathrm{I}_{2}$, Ionization chambers.

is provided with two ionization chambers which are adjustable in volume, and the method makes use of the fact that the sensitivity of the ionization chamber is proportional to its volume. The chambers are therefore so adjusted that one has double the volume of the other, and both are connected in a bridge circuit to the flexible cable of the X-ray dosemeter. The chamber potentials are of opposite signs, so that their wall-electrodes are connected to the plus and minus poles of independent batteries. The inner electrodes are both connected through the highly insulated cable to the needle of a Lindeman electrometer, which is mounted in the measuring apparatus.

If the chambers are simultaneously exposed to $\mathrm{X}$-rays, the electrometer needle moves to one side owing to the greater charge from the larger chamber relative to that of the smaller chamber. In order to obtain an X-ray beam corresponding to a given HVL, say $2 \mathrm{~mm} \mathrm{Cu}$, the larger chamber is covered with this thickness of copper. The kilovoltage of the X-ray unit is then altered until the currents due to the ionization in the two chambers are equal and opposite, and the needle of the electrometer reads zero.

If however, the HVL corresponding to a given $\mathrm{X}$-ray beam is to be determined, the filtration over the larger chamber is altered, until the electrometer needle is in balance. Then the thickness of metal over this chamber corresponds to the HVL of the incident radiation. This latter method is less convenient but rarely required for photographic purposes, as usually the generation of an X-ray beam corresponding to a given HVL is wanted.

\section{Circuit and Apparatus}

Fig. 1 shows the circuit of the actual dosemeter on the left of the dotted lines at $\mathrm{W}_{1}, \mathrm{~W}_{2}$ and that of the two ionization chambers including batteries etc. on the right

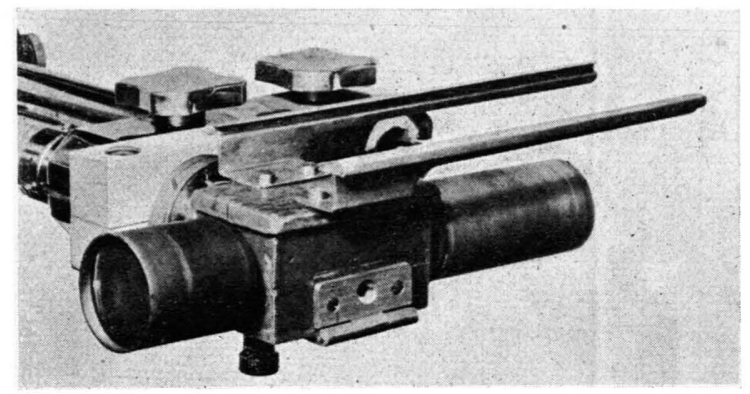

Fig. 2. Junction box with two adjustable ionization chambers and filter supporting device.

of $\mathrm{W}_{1}, \mathrm{~W}_{2}$. Details of the circuit are given in the caption to Fig. 1.

Besides the dosemeter control box the HVL meter apparatus consists of:

(a) The junction box which can be screwed into the terminal of the highly insulated flexible cable and which provides the mechanical and electrical connection of the two chambers with the dosemeter control (see Fig. 2).

(b) The two ionization chambers which are adjustable in volume by movable lids. Their volumes are about 90 and 45 cc respectively.

The chambers are constructed as "thimble chambers“ and are made of „Catalin“, a plastic material. They are 
cylindrical in shape, the walls being $1 \mathrm{~mm}$ thick and coated inside with „Aquadag“, a colloidal graphite. The conductive coating serves as an outer electrode and makes contact with a brass screw in the bottom of the chambers and protruding to the outside. The inner electrode consists of a graphite rod which is held by a small brass bush. The latter is inserted into the bottom but insulated from it by amber. The movable lids consist of discs of catalin which screw into the cylindrical walls so that the volume of the chambers can be adjusted:

(c) Two $54 \mathrm{~V}$ batteries supplying the chamber potential (at saturation).

(d) Two brass rods mounted on the junction box which serve as support for the filters of the larger chamber.

\section{Calibration}

The sensitivity of ionization chambers of similar construction and electrical field distribution is proportional to their volume. In the circuit described, the capacities of the two chambers are added together but the sensitivities of the chambers are still proportional to their volumes.

Some difficulties were encountered in the construction of the chambers to specified dimensions, owing to their thin walls. In order to obtain the sensitivity of 1:2 for the two chambers the following method has been adopted. By exposing the chambers to X-rays, measurements have been made with the 45 cc chamber when the 90 cc chamber was covered with $4 \mathrm{~mm}$ lead, and with the 90 cc chamber whilst the $45 \mathrm{cc}$ chamber was covered with $4 \mathrm{~mm}$ lead. The measurements were carried out by giving definite $\mathrm{X}$-ray doses as measured with a Victoreen X-ray dosemeter. By adjusting the volumes of the chambers by the movable lids, the sensitivity ratio of $1: 2$ could be easily achieved. A check was then made to assure that the sensitivity ratio of $1: 2$ was identical for soft and hard radiation. This condition was fulfilled.

\section{Accuracy and results}

Comparative measurements were made using the HVL meter and the method of succesive absorption measurements. The results illustrated in Fig. 3 show the relationship between $\mathrm{HVL}$ and filtration of a $120 \mathrm{KV}$ X-ray beam as obtained from a $140 \mathrm{KV}$ Siemens-Schuckert X-ray unit (Villard circuit using 1 rectifying valve).

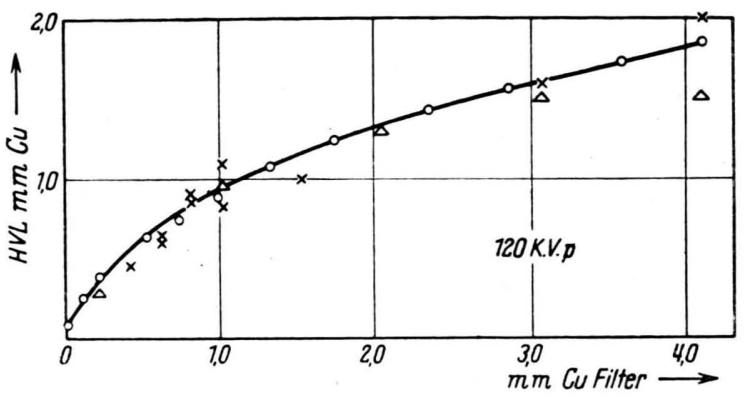

Fig. 3. Relationship between HVL and filtration of an $\mathrm{X}$-ray beam generated at $120 \mathrm{KVp}$ ( $\supset$ values obtained by HVL meter; $x$ values obtained by successive absorption measurements).

The accuracy of the method cannot readily be assessed since there is no more accurate method of measuring HVL's with which it could be compared. The reproducibility of the determination of the HVL in terms of $\mathrm{Cu}$ filter thickness for a given constant voltage was found to be \pm 5 per cent.

The sensitivity of the method depends on the filtration used and cannot be readily expressed, because of the difficulty of measuring very small changes of kilovoltage on the voltmeter of the X-ray control table. It can be said however, that once a balance point of the electrometer needle is reached the measuring system is immediately thrown out of balance by making the smallest voltage adjustment. 